\title{
Application of Functional Endoscopic Sinus Surgery with Navigation system in Medical University - Pleven: Pilot results
}

\author{
Duhlenski B., Valkov Al., Nikolov G., Mirchev St., Stoyanov Tsv., Mladenov Tsv., \\ Kyuchukova-Kazandjieva Kr., Todorova-Doneva J., Yulduz M. \\ Department of Otorhinolaryngology, Medical University - Pleven, Clinic of Otorhinolaryngology, \\ University Hospital „Dr. Georgi Stranski“ - Pleven
}

\begin{abstract}
Background: Functional Endoscopic Sinus Surgery (FESS), supported by a navigation system, is a modern minimally invasive method for diagnosis and surgical treatment of nasal polyposis, acute and chronic sinusitis, benign and malignant tumors of the nasal cavities and skull base, congenital anomalies, severe epistaxis, and a number of other pathological processes in these anatomical areas. Advantages of the method are the improved orientation through navigation, reduced blood loss, sparing of neighboring tissues and structures, optimization of postoperative results, reduction of recovery period and pain as well as the optimized quality of life of the patient.

Aim: Presentation of pilot results from the first surgical interventions under Work Package 3 "Endoscopic sinus surgery, supported by a navigation system, in ENT practice", performed in the "Integrated Interdisciplinary Operating Unit with Navigation and Telesurgery Systems" built under the project BG05M2OP001-1.002-0010 “Center of Competence in Personalized Medicine, 3D and Telemedicine, Robot-assisted and Minimally Invasive Surgery" funded by the "Science and education for smart growth" Operational Program and the European Regional Development Fund.

Results: The surgical interventions were performed without intraoperative and early postoperative complications; the recovery period was smooth. During the follow-up examinations, we observed good local postoperative status, optimal period of healing and absence of subjective complaints reported by the patients.

Conclusion: Our pilot results support the advantages of navigated FESS for safer, more precise and sparing radical treatment of diseases of the nose and nasal cavities. Extended multidisciplinary studies are forthcoming within the project for overall assessment of the significance and applicability of the introduced method in different groups of patients.
\end{abstract}

Keywords: FESS, navigation system, minimally invasive surgery, diseases of the nose and nasal cavities

\section{Background}

Functional endoscopic sinus surgery (FESS), supported by a navigation system, is a minimally invasive method for diagnosis and surgical treatment of inflammatory and infectious diseases of the nose and nasal cavities including nasal polyps, acute and chronic rhinosinusitis, tumors, congenital anomalies and other pathological processes in these anatomical areas. Microdebriders in combination with suction have additionally optimized the removal of pathologic tissue while preserving normal mucosa. [1-9].

The FESS is most commonly used to treat chronic sinusitis that does not respond to standard medical treatment. Patient suffering and quality-of-life impairment with lack of response to conservative treatments may influence the preference of the new method when taking decision about the surgical approach. Among the advantages to FESS over the open sinus procedures are: the improved navigation orientation; reduced blood loss; sparing of adjacent tissues and structures; optimization of postoperative results with pain reduction and recovery period and improved quality of life of the patient [10-12]. Potential adverse events can vary in frequency, severity and time of onset (early and late) from mild intra- and postoperative complications like hyposmia, periorbital ecchymosis, orbital emphysema, epistaxis and adhesions to more severe intraoperative complications with relatively low frequency of $0.3-3.0 \%$ presented by anosmia, nasolacrimal duct 
trauma or carotid injury and, rarely (less than $0.5 \%$ ), severe complications with orbital and intracranial penetration, orbital hematoma, optic nerve damage, visual disturbances and irreversible blindness; the most severe and life-threatening complications appear with cerebrospinal fluid leakage, intracranial hemorrhage, cerebral infarction, pneumoencephalitis, meningitis and other central nervous system complications. The purpose of navigational monitoring during surgery is to connect the images from the preoperative computed tomography (CT) with the anatomy of the treated area, to monitor surgical instruments inside the sinuses with precisely establishing the location of critical structures and organs and to allow performing the surgical intervention with the least possible damage [13-17].

\section{Aim}

We aimed to present the Operating unit technical equipment and the pilot results from the first surgical interventions under the Work Package 3 "Endoscopic sinus surgery, supported by a navigation system, in ENT practice" which were performed in the "Integrated Interdisciplinary Operating Unit with Navigation and Telesurgery Systems" built under the project BG05M2OP001-1.002-0010 "Center of Competence in Personalized Medicine, 3D and Telemedicine, Robot-assisted and Minimally Invasive Surgery" funded by the "Science and education for smart growth" Operational Program and the European Regional Development Fund.

\section{Materials and Methods}

Place of study: Integrated Interdisciplinary Operating Unit with Navigation and Telesurgery Systems in Otorhinolaryngology practice located at the II Base of the University Hospital "Dr. Georgi Stranski" of MU-Pleven. Research and clinical bases: ENT Department, MU- Pleven; ENT Clinic, University Hospital "Dr. Georgi Stranski” Pleven.

Study period: February - November 2020.

Study materials: (1) Ear-nose-throat (ENT) technical equipment and tools: Olympus endoscopic processor OTV-S190, CLV-S190 light source and HD camera head CN-S190; full HD Camera head CH-S190-XZ-E; Olympus Diego ${ }^{\circledR}$ Elite ENT surgery shaver, aspiration module, activation pedal for shaver suction module, and radio frequency generator; Olympus sinuscope, Trueview II optical telescope, mechanically adjustable sinuscope Telescope "V-DOV" and hand tool set; Olympus Celon
Elite ProCut handpiece monopolar cutting handle, monopolar cutting forceps, gripping forceps, electrode for handle for monopolar cutting. (2) Navigation system in an integrated operating room for ENT practice Brainlab Kick 2.0 EM (3) Visualization and documentation: Olympus 32-inch HDTV Medical Monitor FS and connected medical recorder for capture of full HD images and videos. Study population: Male and female patients aged 18-75 and being hospitalized in the Clinic of Otorhinolaryngology at the study clinical base who met the study inclusion criteria and underwent FESS with navigation system during the pilot study period.

Ethical considerations: The research was approved by the Ethic Committee of MU-Pleven in April 2019. A written informed consent was obtained $a$ priory from the study participants.

Inclusion criteria:

- Hospitalized patients, men and women, aged 18-75 years, with indications for surgical treatment of pathological processes of the nose and nasal cavities

- Signed informed consent to participate in the study

- Chronic rhinosinusitis with nasal polyposis

- Histologically proven tumors of the nose and nasal cavities staging T1-3N0M0

- Indications for surgical revision and reconstruction of the nose and nasal cavities

- Computed tomography of the nasal cavities to support the diagnosis

Exclusion criteria:

- Patients aged less than 18 and over 75

- Lack of informed consent to participate in the study

- Refusal to participate in some of the stages of the study

- Contraindications to general anesthesia

- Lesions involving the base of the skull with penetration into the intracranial space

- Large tumors MRI / CT / PET-CT for T4 N1-2M1 distant metastases

- Presence of implanted cochlear devices and / or pacemaker

- Aberrant anatomy of the carotid artery restricting sphenoidal access

Other methods: A comprehensive non-invasive approach to pain assessment in the postoperative period with application of appropriate pain assessment scales such as a 6-point Verbal Rating Scale (VRS) and Visual Analogue Scale (VAS). 


\section{Results}

Performed activities under Stage 1 of the study for the period February-June 2020:

- Delivery, installation and technical testing of FESS with navigation system;

- Equipment with surgical instruments, consumables and specialized devices for endoscopic MIH surgery in ENT practice.

Performed activities under Stage 1 of the study for the period July-August 2020:

- Opening and commissioning of the integrated interdisciplinary operating room with navigation and telesurgery systems in the field of ENT, Oncosurgery and Gynecology at the Department of Minimally Invasive Surgery (MIH) of the Leonardo Da Vinci Competence Center at MU-Pleven.

- On $21^{\text {st }}$ July 2020, the first ENT FESS intervention with navigation was performed.

- The first operated female patient was admitted to the ENT clinic with complaints of unilateral nasal difficulty breathing, loss of smell and periodic one-sided nosebleeds. Anterior rhinoscopy and nasal endoscopy revealed a tumor mass. The FESS intervention with a navigation system has been successfully applied for removal of the tumor from a hard-to-reach and high-risk area. After a three-day hospital stay with a smooth recovery period, the patient was discharged without complications and in good general condition.

Performed activities under Stage 1 of the study for the period September-October 2020:

- A total of 9 patients (5/4 males/females) suffering from chronic rhinosinusitis with/without nasal polyps who failed to respond to conservative treatment ( 7 cases) and tumors of the nose and paranasal sinuses (2 cases) underwent FESS during the pilot study. Along with standard procedures, CT of the paranasal cavities was performed preoperatively to determine the volume of surgical treatment.

- Preoperative time: 8-10 min

- Intraoperative time: 50-90 min

- Intraoperative blood loss: 50-70 ml

- In all patients, the recovery period was smooth and without severe complications. The control examinations reported good local postoperative status, optimal recovery and absence of subjective complaints. There was no recurrence of symptoms clinically observed in any of the patients by up-to-date follow-up. None of the patients required revision surgery in the first month after operation. Patients were generally satisfied with the first results after operation.

- The team work process and visualization of the performed surgical interventions documented in the course of endoscopic surgical treatment are presented in Figures 1-6.

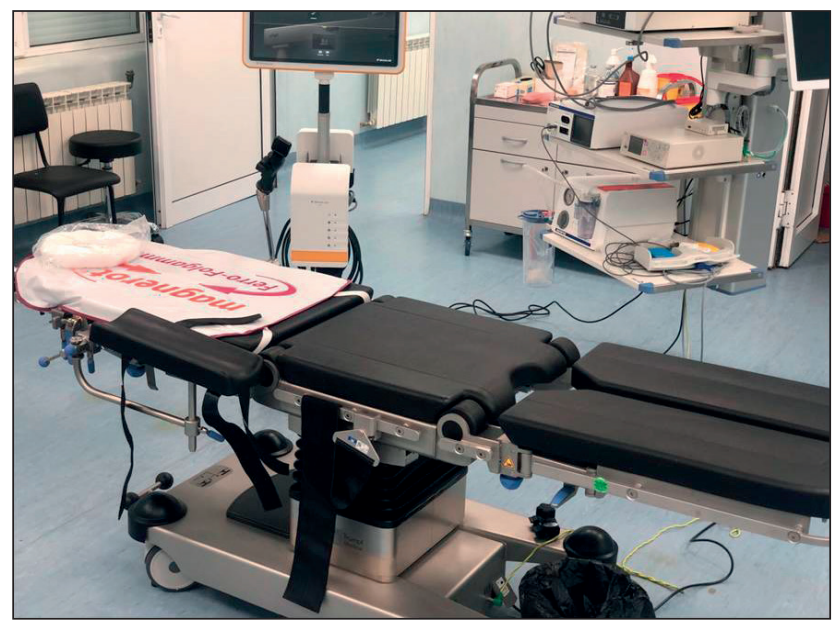

Figure 1. The operating room equipped for FESS with navigation system.
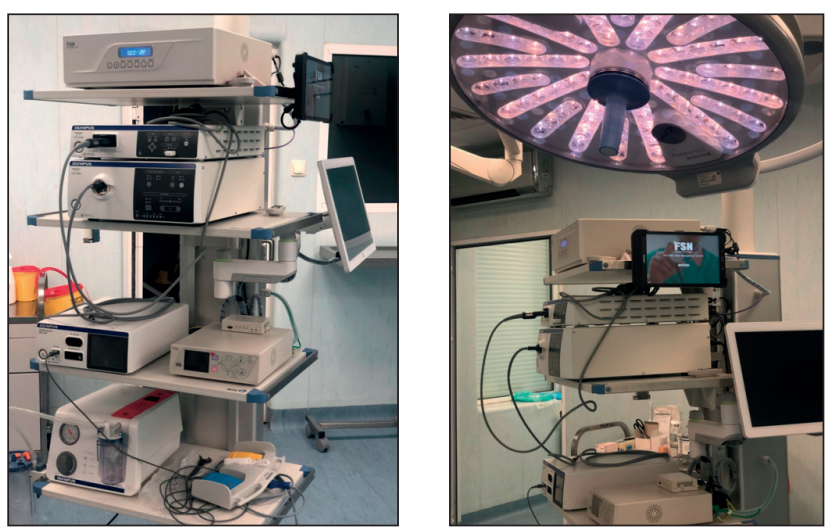

Figure 2. The systems for visualization and documentation in the operating room.

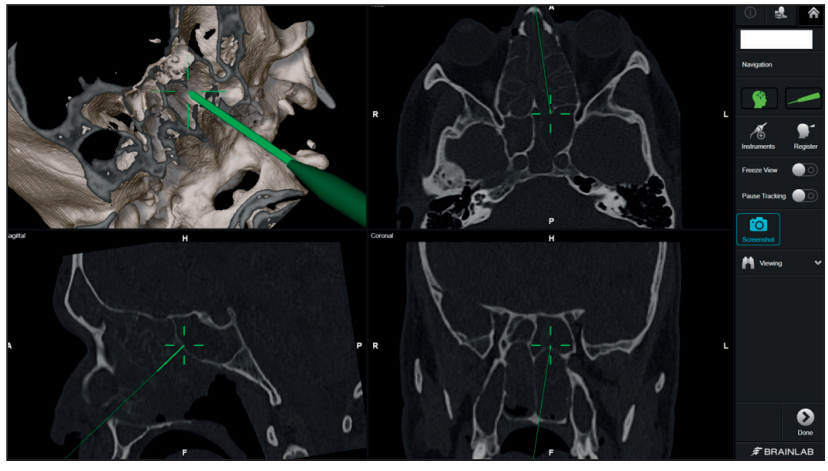

Figure 3. Demonstration of the navigation system in FESS surgery. 


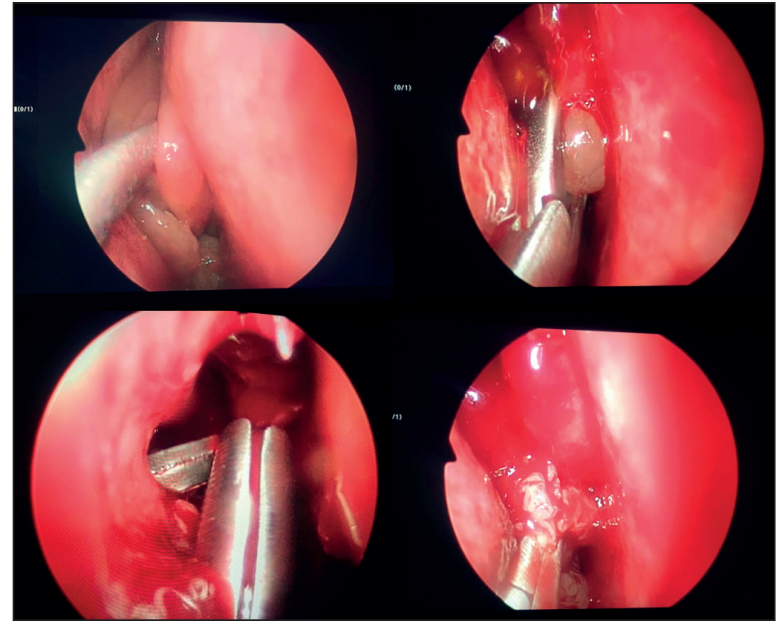

Figure 4. Demonstration of the intra-operative process visualization during FESS surgery.

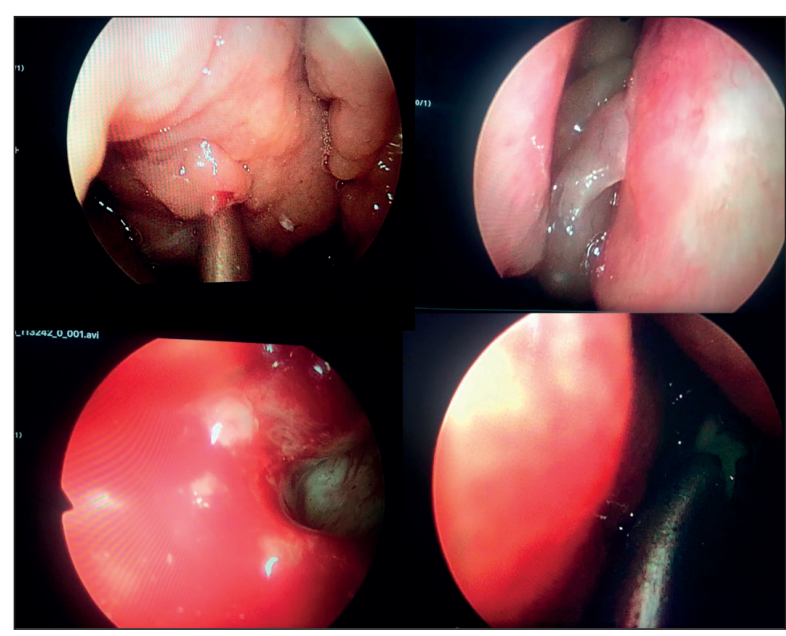

Figure 5. Demonstration of the intra-operative process visualization during FESS surgery.

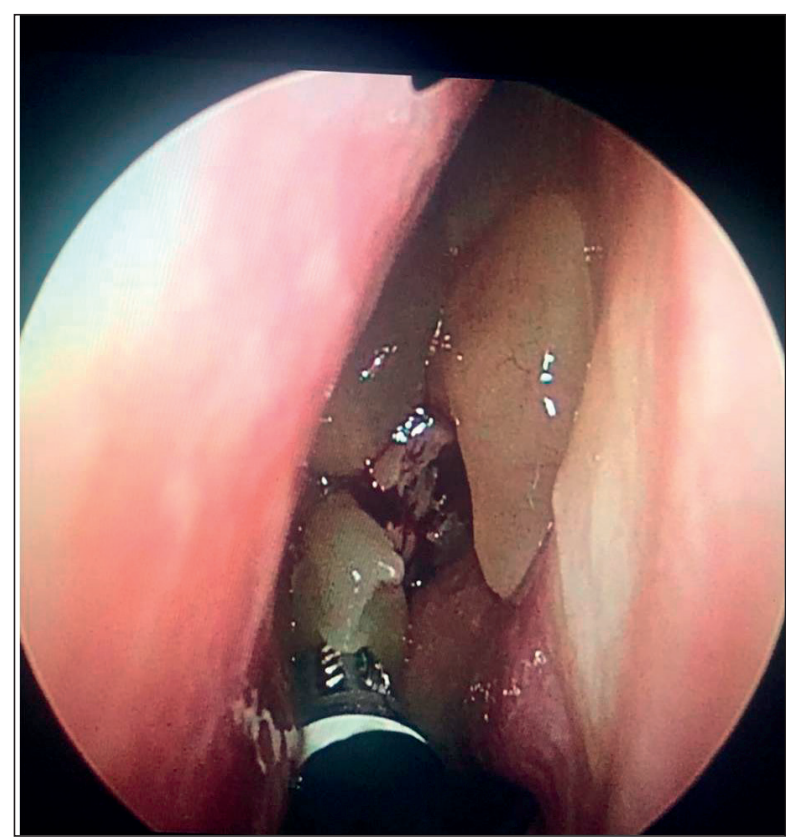

Figure 6. Demonstration of the intra-operative process visualization during FESS surgery.

\section{Discussion}

Our study demonstrates the first regional research on the use of FESS with navigation system in the ENT practice. The Integrated Interdisciplinary Operating Unit with Navigation and Telesurgery Systems is the latest commissioned high-technological and modern base for interdisciplinary minimal invasive surgery. It is also one of the few national university centers which developed and introduced the FESS in ENT surgery.

Our initial findings on the use of FESS with navigation system correspond to the publications of national researchers during the last decade [18-20]. With the first stage of our project, a new promising field has been opened for national collaboration with university centers of similar high technology equipment and competence activities in advanced ENT surgery from Sofia, Varna, Plovdiv and Stara Zagora.

\section{Conclusion}

The endoscopic sinus surgery FESS combined with the navigation system is characterized by millimeter precision and is significantly gentler than conventional open techniques, in which external incisions disrupt tissue integrity, prolong the recovery period and often leave unsightly scars.

The navigation system improves the surgeon's orientation and accuracy during work and gives a clearer personalized idea of the characteristics of the lesion and the location of important adjacent structures and blood vessels. The combination of FESS with a navigation system reduces intraoperative bleeding, protects the surrounding tissues and optimizes postoperative results by shortening the recovery period, reducing postoperative pain and improving the patient's quality of life.

Our pilot results support the advantages of FESS with a navigation system for safer, more precise and gentle radical treatment of diseases of the nose and nasal cavities. Extended multidisciplinary studies are forthcoming within the project for overall assessment of the significance and applicability of the introduced minimal invasive method in ENT practice.

This study was presented online at the XIV National Congress of Otorhinolaryngology, 20-21 November 2020, Bulgaria.

The published results are a part of the activities under the Project BG05M2OP001-1.002-0010 CENTER OF COMPETENCE IN PERSONALISED MEDICINE, 3D AND TELEMEDICINE, ROBOT-ASSISTED AND MINIMALLY INVASIVE SURGERY funded by the "Science and Education for Smart Growth" Operational Program and the European Regional Development Fund. 


\title{
References:
}

1. Chu ST. Endoscopic sinus surgery under navigation system - analysis report of 79 cases. J Chin Med Assoc 2006; 69(11): 529-533

2. Strauss G, Koulechov K, Roettger S; Bahner J; Trantakis C, Hofer M, Korb W, et al. Evaluation of Navigation System for ENT. Laryngoscope 2006; 116: 564-572

3. Smith T, Stewart MG, Orlandi RR, Setzen M, Lanza DC. Indications for image-guided sinus surgery: the current evidence. Am J Rhinol 2007; 21(1): 80-83

4. Christopher Goh HK, Ng YH, Wei Teo DT. Minimally invasive surgery for head and neck cancer. Lancet Oncol 2010; 11: 281-286

5. Ivan ME, Jahangiri A, El-Sayed IH, Aghi MK. Minimally invasive approaches to the anterior skull base. Neurosurg Clin North Am 2013; 24(1): 19-37

6. Stanford Otolaryngology - Head \& Neck Surgery. Available at: http://med.stanford.edu/ohns

7. Samarakkody ZM, Abdullah B. The use of image guided navigational trackingsystems for endoscopic sinus surgery and skull base surgery: A review. EJENTAS 2016; 17: 133-137

8. Leonard S, Reiter A, Sinha A, Ishii M, Taylor RH, Hager GD. Image-based navigation for Functional Endoscopic Sinus Surgery using structure from motion. Proc SPIE Int Soc Opt Eng. 2016; 9784: doi:10.1117/12.2217279

9. Siddaiah-Subramanya M, Tiang KW, Nyandowe M. A new era of minimally invasive surgery: A review of progress and development of major technical innovations in the last decade. SM Min Inv Surg. 2017; 1(1): 1002

10. Reddy MS, Reddy CRVB.Effectiveness of minimally invasive endoscopic sinus surgery in alleviating the symptom complex in chronic rhinosinusitis. Int J Otorhinolaryngol Head Neck Surg 2018; 4: 517-521

11. Vivek B. Role of endoscopy in the management of benign and malignant sinonasal tumors. Dissertation 2018. Upgraded Institute of Otorhinolaryngology. Madras Medical College, Chennai, Tamilnadu, India.

12. Tomazic V. Quo vadis FESS? Future directions in functional endoscopic sinus surgery. ENT \& AUDIOLOGY NEWS $2019 ; 28$ (2): 40-42.

13. Nicolai P, Bradley PJ. Anterior skull base tumors. Adv Otorhinolaryngol 2020; 84: 56-67

14. Nogueira JF, de Sousa Lobo Ferreira Querido R, Gonçalves da Silva Leite J, Cabral da Costa T. Future of Endoscopic Ear Surgery. Otolaryngologic Clinics of North America 2020. Article in press. doi:10.1016/j.otc.2020.09.023

15. Singh A, Kumar R, Thakar A, Sharma SC, Bhalla AS. Role of image guided navigation in endoscopic surgery of paranasal sinuses: A comparative study. Indian J Otolaryngol Head Neck Surg 2020; 72: 221-227

16. Schmale IL, Vandelaar LJ, Luong AU, Citardi MJ, Yao WC. Image-guided surgery and intraoperative imaging in rhinology: Clinical update and current state of the art. Ear, Nose \& Throat Journal 2020; 1-12

17. Linxweiler M, Pillong L, Kopanja D, Kuhn JP, Wagenpfeil S, et al. Augmented reality-enhanced navigation in endoscopic sinus surgery: A prospective, randomized, controlled clinical trial. Laryngoscope Investigative Otolaryngology 2020; 5: 621-629

18. Tsvetkov V, Zlatanov, Kolev, Kukushev G. Functional Endoscopic Sinus Surgery (FESS) The gold standard in the treatment of impaired nasal breathing and the diseases of paranasal cavities: 5-year review of 300 operations (Original article published in Bulgarian). MEDINFO 2011; 3: 16-17

19. Benchev R. Rhinosinusitis and Functional Endoscopic Sinus Surgery. Available at: http://rumenbenchev.com/ear-nose-and-throat/ rhinosinusitis/2014

20. Kukushev G. Chronic rhinosunusitis with nasal polyps in adults. Monograph 2020 (Published in Bulgarian); Sofia. ISBN 978-619-188518-3

\author{
Correspondence to: \\ Boris Duhlenski, MD \\ Clinic of Otorhinolaryngology \\ University Hospital "Dr. Georgi Stranski"-Pleven \\ II Clinical Base, 91 Gen. Vladimir Vazov Str. \\ Pleven 5800 \\ Email: duhlenski_b@abv.bg \\ Mobile phone: $+3 \overline{59886804296}$
}

